\title{
Nonnegativity, Reducibility, and SemiStability of Mass Action Kinetics
}

\author{
Dennis S. Bernstein \\ Department of Aerospace Engineering \\ The University of Michigan \\ Ann Arbor, MI 48109-2140 \\ dsbaero@engin. umich.edu
}

\section{Introduction}

Mass action kinetics are used throughout the chemical and chemical engineering literature to describe the dynamics of systems of chemical reaction laws. They are also a special form of compartmental systems that involve mass balance relations [1]. Aside from their obvious importance in chemical engineering applications, mass action kinetics have numerous analytical properties that are of inherent interest from a dynamical systems perspective.

In Section 2 we provide a general construction of the kinetic equation from reaction laws based upon the formulation given in [2]. In Section 3 we consider the nonnegativity of the solutions to the kinetic equation. Next, in Section 4, we consider the reducibility of the mass action kinetics. In Section 5 we consider the stability of the equilibria of the kinetic equation. To do this, we apply Lyapunov methods to the kinetic equation and obtain results that guarantee semistability, that is, convergence to an equilibrium that depends upon initial concentrations. This notion is developed in [4], which extends the linear semistability theory of [5] to nonlinear systems. In Section 6 we revisit the remarkable "zero deficiency" result of [3], which provides rate-independent conditions guaranteeing stability.

A vector $x \in \mathcal{R}^{p}=\mathcal{R}^{p \times 1}$ is a $p \times 1$ column vector, while the set of $p \times q$ real matrices is denoted by $\mathcal{R}^{p \times q}$. For $x \in$ $\mathcal{R}^{p}$ we write $x \geq \geq 0$ to indicate that every component of $x$ is nonnegative and $x>0$ to indicate that every component of $x$ is positive. In this case we say that $x$ is nonnegative or positive, respectively. Likewise, $A \in$ $\mathcal{R}^{p \times q}$ is nonnegative or positive if every entry of $A$ is nonnegative or positive, respectively, which is written as $A \geq \geq 0$ or $A>>0$, respectively. Let $\overline{\mathcal{R}}_{+}^{n}$ and $\mathcal{R}_{+}^{n}$

\footnotetext{
${ }^{1}$ This research was supported in part by the Air Force Office of Scientific Research under grant F49620-98-1-0019.
}

Sanjay P. Bhat

Department of Aerospace Engineering

Indian Institute of Technology

Powai, Mumbai 400076, India

bhatQaero.iitb.ernet.in

denote the nonnegative and positive orthants of $\mathcal{R}^{n}$, respectively, that is, if $x \in \mathcal{R}^{n}$, then $x \in \overline{\mathcal{R}}_{+}^{n}$ and $x \in$ $\mathcal{R}_{+}^{n}$ are equivalent, respectively, to $x \geq \geq 0$ and $x>>0$.

For vectors $x, y \in \mathcal{R}^{p}$ or matrices $A, B \in \mathcal{R}^{p \times q}$ we use $x \circ y$ and $A \circ B$ to denote component-by-component and entry-by-entry multiplication, respectively. The $p \times p$ identity matrix is written as $I_{p}$. The transposes of $x \in \mathcal{R}^{p}$ and $A \in \mathcal{R}^{p \times q}$ are denoted by $x^{\mathrm{T}}$ and $A^{\mathrm{T}}$, respectively. For a matrix $A \in \mathcal{R}^{p \times q}$ let $\operatorname{row}_{i}(A)$ and $\operatorname{col}_{j}(A)$ denote the $i$ th row and $j$ th column of $A$, respectively. For $x \in \mathcal{R}^{q}$ and nonnegative $A=\left[A_{i j}\right] \in \mathcal{R}^{p \times q}$, $x^{A}$ denotes the element of $\mathcal{R}^{p}$ whose ith component for $i=1, \ldots, p$ is the product $x_{1}^{A_{i 1}} \cdots x_{q}^{A_{i q}}$. We define $0^{0}=1$.

\section{Kinetic Equation}

Consider $s$ species $x_{1}, \ldots, x_{s}$, where $s \geq 1$, whose interactions are governed by $r$ reactions, where $r \geq 1$, comprising the reaction network

$$
\sum_{j=1}^{s} A_{i j} x_{j} \stackrel{k_{i}}{\longrightarrow} \sum_{j=1}^{s} B_{i j} x_{j}, \quad i=1, \ldots, r,
$$

where, for $i=1, \ldots, r, k_{i}>0$ is the reaction rate of the $i$ th reaction, $\sum_{j=1}^{s} A_{i j} x_{j}$ is the reactant of the $i$ th reaction, and $\sum_{j=1}^{s} B_{i j} x_{j}$ is the product of the $i$ th reaction. Note that each reaction in the reaction network (2.1) is represented as being irreversible. However, reversible reactions can be modeled by including the reverse reaction as a separate reaction. Each stoichiometric coefficient $A_{i j}$ and $B_{i j}$ is assumed to be a nonnegative number which is usually, but not necessarily, an integer. The reaction network (2.1) can be written compactly in matrix-vector form as

$$
A x \stackrel{k}{\longrightarrow} B x,
$$

where $x \in \mathcal{R}^{s}$ and $k \in \mathcal{R}^{r}$ denote the column vectors $\left[x_{1} \cdots x_{s}\right]^{\mathrm{T}}$ and $\left[k_{1} \cdots k_{r}\right]^{\mathrm{T}}$, respectively, and $A$ and $B$ 
denote the $r \times s$ nonnegative matrices $A=\left[A_{i j}\right]$ and $B=\left[B_{i j}\right]$.

Next, with a slight abuse of notation, we let $x_{j}=x_{j}(t)$, $j=1, \ldots, s$, denote the concentration of the species $x_{j}$ at time $t$. Then the dynamics of the reaction network (2.2) are assumed to be given by mass action kinetics, whose dynamics are modeled by the kinetic equation

$$
\dot{x}(t)=(B-A)^{\mathrm{T}}\left[k \circ x^{A}(t)\right], \quad t \geq 0 .
$$

Defining $K \triangleq \operatorname{diag}\left(k_{1}, \ldots, k_{r}\right)$, (2.3) can be written as

$$
\dot{x}(t)=(B-A)^{\mathrm{T}} K x^{A}(t), \quad t \geq 0 .
$$

In mass action kinetics the reaction order $\sum_{j=1}^{s} A_{i j}$ of the $i$ th reaction is the sum of the stoichiometric coefficients of the species appearing in the reactant of the $i$ th reaction. It can be seen that the kinetic equation (2.3) is linear if and only if each row of $A$ contains exactly one 1 with the remaining entries equal to zero, that is, if and only if each reaction is unimolecular. In this case (2.3) becomes

$$
\dot{x}(t)=M x(t), \quad t \geq 0,
$$

where $M \in \mathcal{R}^{s \times s}$ is defined by

$$
M \triangleq(B-A)^{\mathrm{T}} K A
$$

The reaction network (2.2) is not limited to closed systems for which conservation of mass holds. In fact, (2.2) can also be used to represent open systems in which mass removal and mass addition are allowed. For example, the reactions $x_{1} \stackrel{k_{3}}{\rightarrow} 0$ and $0 \stackrel{k_{1}}{\rightarrow} x_{1}$ are both allowed, where the reactants are $x_{1}$ and 0 , respectively, and the products are 0 and $x_{1}$, respectively. The kinetic equations for these reactions, which represent the removal and addition of mass, respectively, are $\dot{x}_{1}(t)=-k_{1} x_{1}(t)$ and $\dot{x}_{1}(t)=k_{1}$ with solutions $x_{1}(t)=x_{1}(0) e^{-k_{1} t}$ and $x_{1}(t)=k_{1} t+x_{1}(0)$, respectively. The reactions $x_{1} \stackrel{k_{1}}{\rightarrow} 2 x_{1}$ and $2 x_{1} \stackrel{k_{1}}{\rightarrow} 3 x_{1}$, which also represent the addition of mass, have the kinetics $\dot{x}_{1}(t)=k_{1} x_{1}(t)$ and $\dot{x}_{1}(t)=k_{1} x_{1}^{2}(t)$ with solutions $x_{1}(t)=x_{1}(0) e^{k_{1} t}$ and $x_{1}(t)=x_{1}(0) /\left(1-k_{1} x_{1}(0) t\right)$, respectively.

Example 1. Consider the reaction network

$$
\begin{array}{lll}
x_{1} & \stackrel{k_{3}}{\rightarrow} & x_{2}, \\
x_{2} & \stackrel{k_{2}}{\rightarrow} & x_{1},
\end{array}
$$

so that $s=2, r=2$, and $A$ and $B$ are given by

$$
A=\left[\begin{array}{ll}
1 & 0 \\
0 & 1
\end{array}\right], \quad B=\left[\begin{array}{ll}
0 & 1 \\
1 & 0
\end{array}\right] .
$$

The kinetic equations are thus given by

$$
\begin{aligned}
& \dot{x}_{1}=-k_{1} x_{1}+k_{2} x_{2}, \\
& \dot{x}_{2}=k_{1} x_{1}-k_{2} x_{2},
\end{aligned}
$$

or in linear system form by (2.5) where

$$
M=\left[\begin{array}{rr}
-k_{1} & k_{2} \\
k_{1} & -k_{2}
\end{array}\right] .
$$

Example 2. Consider the reaction network

$$
\begin{array}{rll}
x_{1}+x_{2} & \stackrel{k_{3}}{\rightarrow} 2 x_{1}, \\
2 x_{1} & \stackrel{k_{2}}{\rightarrow} & x_{1}+x_{2},
\end{array}
$$

so that $s=2, r=2$,

$$
A=\left[\begin{array}{ll}
1 & 1 \\
2 & 0
\end{array}\right], \quad B=\left[\begin{array}{ll}
2 & 0 \\
1 & 1
\end{array}\right] \text {. }
$$

The kinetic equations are thus given by

$$
\begin{aligned}
& \dot{x}_{1}=k_{1} x_{1} x_{2}-k_{2} x_{1}^{2}, \\
& \dot{x}_{2}=-k_{1} x_{1} x_{2}+k_{2} x_{1}^{2} .
\end{aligned}
$$

Example 3. The Lotka-Volterra reaction is given by

$$
\begin{array}{rll}
x_{1} & \stackrel{k_{3}}{\rightarrow} 2 x_{1}, \\
x_{1}+x_{2} & \stackrel{k_{2}}{\rightarrow} 2 x_{2}, \\
x_{2} & \stackrel{k_{3}}{\rightarrow} 0,
\end{array}
$$

where $x_{1}$ and $x_{2}$ denote prey and predator concentrations, respectively, so that $s=2$ and $r=3$. Furthermore, $A$ and $B$ are given by

$$
A=\left[\begin{array}{ll}
1 & 0 \\
1 & 1 \\
0 & 1
\end{array}\right], \quad B=\left[\begin{array}{ll}
2 & 0 \\
0 & 2 \\
0 & 0
\end{array}\right]
$$

Consequently, the kinetic equations have the form

$$
\begin{aligned}
& \dot{x}_{1}=k_{1} x_{1}-k_{2} x_{1} x_{2}, \\
& \dot{x}_{2}=-k_{3} x_{2}+k_{2} x_{1} x_{2} .
\end{aligned}
$$

Example 4. A widely studied reaction network involves the interaction of a substrate $S$ and an enzyme $E$ to produce a product $P$ via an intermediate species C. The reactions are given by

$$
\mathrm{S}+\mathrm{E} \underset{k_{2}}{\stackrel{k_{1}}{\rightleftharpoons}} \mathrm{C} \stackrel{k_{3}}{\rightarrow} \mathrm{P}+\mathrm{E}
$$

so that $s=4$ and $r=3$. Letting $x_{1}=\mathrm{S}, x_{2}=\mathrm{C}, x_{3}=$ $\mathrm{E}$, and $x_{4}=\mathrm{P}$, the corresponding reaction network can 
be written as

$$
\begin{array}{rll}
x_{1}+x_{3} & \stackrel{k_{1}}{\rightarrow} x_{2}, \\
x_{2} & \stackrel{k_{2}}{\rightarrow} x_{1}+x_{3}, \\
x_{2} & \stackrel{k_{3}}{\rightarrow} & x_{3}+x_{4} .
\end{array}
$$

It thus follows that $A$ and $B$ are given by

$$
A=\left[\begin{array}{llll}
1 & 0 & 1 & 0 \\
0 & 1 & 0 & 0 \\
0 & 1 & 0 & 0
\end{array}\right], \quad B=\left[\begin{array}{llll}
0 & 1 & 0 & 0 \\
1 & 0 & 1 & 0 \\
0 & 0 & 1 & 1
\end{array}\right]
$$

Consequently, the kinetic equations have the form

$$
\begin{aligned}
& \dot{x}_{1}=k_{2} x_{2}-k_{1} x_{1} x_{3}, \\
& \dot{x}_{2}=-\left(k_{2}+k_{3}\right) x_{2}+k_{1} x_{1} x_{3}, \\
& \dot{x}_{3}=\left(k_{2}+k_{3}\right) x_{2}-k_{1} x_{1} x_{3}, \\
& \dot{x}_{4}=k_{3} x_{2} .
\end{aligned}
$$

\section{Nonnegativity}

Definition 3.1. Let $f=\left(f_{1}, \ldots, f_{n}\right): \mathcal{D} \rightarrow \mathcal{R}^{n}$ where $\mathcal{D}$ is an open subset of $\mathcal{R}^{n}$ that contains $\overline{\mathcal{R}}_{+}^{n}$. Then $f$ is essentially nonnegative if, for all $i=1, \ldots, n, f_{i}(x) \geq 0$ for all $x=\left(x_{1}, \ldots, x_{n}\right) \in \overline{\mathcal{R}}_{+}^{n}$ such that $x_{i}=0$.

It is easy to see that if $f$ is the linear function $f(x)=$ $M x$, where $M \in \mathcal{R}^{n \times n}$, then $f$ is essentially nonnegative if and only if all of the off-diagonal entries of $M$ are nonnegative. In this case we say that $M$ is essentially nonnegative.

For the following definitions and results we consider the system

$$
\dot{x}(t)=f(x(t)), \quad x(0)=x_{0},
$$

where $f: \mathcal{D} \rightarrow \mathcal{R}^{n}$ is locally Lipschitz and $\mathcal{D}$ is an open subset of $\mathcal{R}^{n}$. A subset $\mathcal{U} \subseteq \mathcal{D}$ is an invariant set for (3.1) if $x(0) \in \mathcal{U}$ implies that $x(t) \in \mathcal{U}$ for all $t \in\left[0, T_{x(0)}\right)$, where $0<T_{x(0)} \leq \infty$ and $\left[0, T_{x(0)}\right)$ is the maximal interval of existence for the solution $x(\cdot)$ of (3.1).

Theorem 3.1. Suppose that $\overline{\mathcal{R}}_{+}^{n} \subset \mathcal{D}$. Then $\overline{\mathcal{R}}_{+}^{n}$ is an invariant set for (3.1) if and only if $f$ is essentially nonnegative.

Proposition 3.1. Define $f: \mathcal{R}^{s} \rightarrow \mathcal{R}^{s}$ by $f(x)=$ $(B-A)^{\mathbf{T}}\left(k \circ x^{A}\right)$. Then $f$ is essentially nonnegative.

Consider the case in which (2.3) is linear so that the kinetic equation is given by (2.5), where $M=B^{\mathrm{T}} K A-$ $A^{\mathrm{T}} K A$. Since $B^{\mathrm{T}} K A$ and $A^{\mathrm{T}} K A$ are nonnegative and since $A^{\mathrm{T}} K A$ is diagonal, it can be seen directly that $M$ is essentially nonnegative.
Theorem 3.2. $\overline{\mathcal{R}}_{+}^{n}$ is an invariant set for (2.3).

\section{Reducibility}

In this section we provide a technique for reducing the number of kinetic equations needed to model the dynamics of the reaction network (2.2). This technique is based upon the fact that, while $x(t)$ is confined to the nonnegative orthant for nonnegative initial conditions, the structure of the kinetic equation (2.3) imposes an additional constraint on the allowable trajectories. To state this result we define the stoichiometric subspace $\mathcal{S}$ by $\mathcal{S} \triangleq \operatorname{Im}\left((B-A)^{\mathrm{T}}\right)$, which is a subspace of $\mathcal{R}^{s}$. The dimension of this subspace is given by $q \triangleq \operatorname{rank}\left((B-A)^{\mathrm{T}}\right)=\operatorname{rank}(B-A)$, which is the rank of the reaction network. The following result shows that the solution of the kinetic equation (2.3) is confined to an affine subspace which is parallel to the stoichiometric subspace. For convenience we let $P \in \mathcal{R}^{s \times s}$ denote the unique orthogonal projector whose range is $\mathcal{S}$, and define $P_{\perp} \triangleq I_{s}-P$. In terms of the generalized inverse $(\cdot)^{+}, P$ is given by $P=(B-A)^{\mathrm{T}}\left[(B-A)^{\mathrm{T}}\right]^{+}=(B-A)^{+}(B-A)$.

Proposition 4.1. Suppose that $x(0)$ is nonnegative. Then the solution $x(\cdot)$ of (2.3) satisfies

$$
x(t) \in(x(0)+\mathcal{S}) \cap \overline{\mathcal{R}}_{+}^{s}
$$

for all $t \in\left[0, T_{x(0)}\right)$.

Corollary 4.1. Suppose that $x(0)$ is nonnegative. Then $(x(0)+\mathcal{S}) \cap \overline{\mathcal{R}}_{+}^{s}$ is an invariant set for (2.3).

Proposition 4.1 shows that the solution $x(\cdot)$ of the kinetic equation (2.3) is confined to the stoichiometric compatibility class $(x(0)+\mathcal{S}) \cap \overline{\mathcal{R}}_{+}^{s}$, which is a $q-$ dimensional manifold. (The set $(x(0)+\mathcal{S}) \cap \mathcal{R}_{+}^{s}$ is a positive stoichiometric compatibility class.) This fact suggests that the dynamics of the system can be represented by a reduced set of $q$ variables. The following result assumes that the species $x_{1}, \ldots, x_{s}$ have been renumbered so that the first $q$ columns of $B-A$ are linearly independent.

Proposition 4.2. Assume that $q<s$. Furthermore, partition $A=\left[\begin{array}{ll}A_{1} & A_{2}\end{array}\right]$ and $B=\left[\begin{array}{ll}B_{1} & B_{2}\end{array}\right]$, where $A_{1}, B_{1} \in \mathcal{R}^{r \times q}$, and assume that $\operatorname{rank}\left(B_{1}-A_{1}\right)=q$. In addition, let $F \in \mathcal{R}^{q \times(s-q)}$ satisfy $A_{2}-B_{2}=$ $\left(A_{1}-B_{1}\right) F$. Finally, partition $x=\left[\begin{array}{ll}\hat{x}_{1}^{\mathrm{T}} & \hat{x}_{2}^{\mathrm{T}}\end{array}\right]^{\mathrm{T}}$, where $\hat{x}_{1} \triangleq\left[x_{1} \cdots x_{q}\right]^{\mathrm{T}}$ and $\hat{x}_{2} \triangleq\left[x_{q+1} \cdots x_{s}\right]^{\mathrm{T}}$. Then

$$
\hat{x}_{2}(t)=F^{\mathrm{T}} \hat{x}_{1}(t)+\gamma, \quad t \geq 0,
$$


where $\gamma \triangleq \hat{x}_{2}(0)-F^{\mathrm{T}} \hat{x}_{1}(0) \in \mathcal{R}^{s-q}$, and $\hat{x}_{1}(\cdot)$ satisfies $\dot{\hat{x}}_{1}(t)=\left(B_{1}-A_{1}\right)^{\mathrm{T}}\left[k \circ \hat{x}_{1}^{A_{1}}(t) \circ\left(F^{\mathrm{T}} \hat{x}_{1}(t)+\gamma\right)^{A_{2}}\right], \quad t \geq 0$.

Example 1 continued. Since $s=2$ and $r=2$ it can be seen that $q=1<s$ so that Proposition 4.2 can be applied with $F=-1$. It thus follows that $x_{2}=$ $-x_{1}+\gamma$ for all $t \geq 0$, where $\gamma=x_{1}(0)+x_{2}(0)$. Applying Proposition 4.2 , we obtain the scalar kinetic equation

$$
\dot{x}_{1}=-\left(k_{1}+k_{2}\right) x_{1}+k_{2} \gamma \text {. }
$$

Example 2 continued. Since $s=2$ and $r=2$ it can be seen that $q=1<s$ so that Proposition 4.2 can be applied with $F=-1$. It thus follows that $x_{2}=$ $-x_{1}+\gamma$ for all $t \geq 0$, where $\gamma=x_{1}(0)+x_{2}(0)$. Applying Proposition 4.2, we obtain the scalar kinetic equation

$$
\dot{x}_{1}=-\left(k_{1}+k_{2}\right) x_{1}^{2}+k_{1} \gamma x_{1} .
$$

Example 3 continued. Since $s=2$ and $r=3$, it can be seen that $q=2=s$ and thus reduction is not possible.

Example 4 continued. Since $s=4$ and $r=3$ it can be seen that $q=2<s$ so that Proposition 4.2 can be applied with $F=\left[\begin{array}{rr}0 & -1 \\ -1 & -1\end{array}\right]$. It thus follows that $x_{3}=-x_{2}+\gamma_{1}$ and $x_{4}=-x_{1}-x_{2}+\gamma_{2}$ for all $t \geq 0$, where $\gamma_{1}=x_{2}(0)+x_{3}(0)$ and $\gamma_{2}=x_{1}(0)+x_{2}(0)+x_{4}(0)$. Applying Proposition 4.2, we obtain

$$
\begin{aligned}
& \dot{x}_{1}=-k_{1} \gamma_{1} x_{1}+k_{2} x_{2}+k_{1} x_{1} x_{2}, \\
& \dot{x}_{2}=k_{1} \gamma_{1} x_{1}-\left(k_{2}+k_{3}\right) x_{2}-k_{1} x_{1} x_{2} .
\end{aligned}
$$

\section{Stability Analysis}

Consider the system

$$
\dot{x}(t)=f(x(t)), \quad x(0)=x_{0},
$$

where $f: \mathcal{D} \rightarrow \mathcal{R}^{n}$ is locally Lipschitz and $\mathcal{D} \subseteq \mathcal{R}^{n}$ is open. We assume that, for all $x_{0} \in \mathcal{D},(5.1)$ has a unique solution on $[0, \alpha)$ for all $\alpha>0$. If $x_{\mathrm{e}} \in \mathcal{D}$ satisfies $f\left(x_{\mathrm{e}}\right)=0$, then $x_{\mathrm{e}}$ is an equilibrium of (5.1).

Definition 5.1. Let $U \subseteq \mathcal{D}$ be invariant with respect to (5.1) and let $x_{\mathrm{e}} \in \mathcal{U}$ be an equilibrium of (5.1). Then $x_{\mathrm{e}}$ is Lyapunov stable with respect to $\mathcal{U}$ if, for every relatively open subset $\mathcal{U}_{\varepsilon}$ of $\mathcal{U}$ containing $x_{\mathrm{e}}$ there exists a relatively open subset $\mathcal{U}_{\delta}$ of $\mathcal{U}$ containing $x_{\mathbf{e}}$ such that, if $x(0) \in \mathcal{U}_{\delta}$, then the solution $x(\cdot)$ of (5.1) satisfies $x(t) \in \mathcal{U}_{\varepsilon}$ for all $t \in[0, \infty)$. Furthermore, (5.1) is semistable with respect to $\mathcal{U}$ if $\mathcal{U}$ is closed, $\lim _{t \rightarrow \infty} x(t)$ exists for all $x(0) \in \mathcal{U}$, and every equilibrium in $\mathcal{U}$ is Lyapunov stable with respect to $\mathcal{U}$. In addition, $x_{\mathrm{e}}$ is asymptotically stable with respect to $\mathcal{U}$ if $x_{\mathrm{e}}$ is Lyapunov stable with respect to $\mathcal{U}$ and there exists a relatively open subset $\mathcal{U}_{\delta}$ of $\mathcal{U}$ containing $x_{\mathrm{e}}$ such that, if $x(0) \in \mathcal{U}_{\delta}$, then $\lim _{t \rightarrow \infty} x(t)=x_{\mathrm{e}}$. Finally, $x_{\mathrm{e}}$ is globally asymptotically stable with respect to $\mathcal{U}$ if the previous statement holds with $\mathcal{U}_{\delta}=\mathcal{U}$.

Definition 5.2. A vector $x_{\mathrm{e}} \in \overline{\mathcal{R}}_{+}^{s}$ satisfying ( $B-$ $A)^{\mathrm{T}}\left(k \circ x_{\mathrm{e}}^{A}\right)=0$ is an equilibrium of (2.3). If, in addition, $x_{\mathrm{e}} \in \mathcal{R}_{+}^{s}$ then $x_{\mathrm{e}}$ is a positive equilibrium of (2.3).

Let $\mathcal{E}$ denote the set of equilibria of $(2.3)$, and let $\mathcal{E}_{+} \subseteq$ $\mathcal{E}$ denote the set of positive equilibria.

Example 1 continued. For this example $\mathcal{E}=$ $\left\{\left(x_{1}, x_{2}\right) \in \overline{\mathcal{R}}_{+}^{2}: x_{2}=\left(k_{1} / k_{2}\right) x_{1}\right\}$.

Example 2 continued. For this example $\mathcal{E}=$ $\left\{\left(x_{1}, x_{2}\right) \in \overline{\mathcal{R}}_{+}^{2}: x_{1}=0\right.$ or $\left.x_{2}=\left(k_{2} / k_{1}\right) x_{1}\right\}$. For the reduced system (4.5) $\mathcal{E}=\left\{0, k_{1} \gamma /\left(k_{1}+k_{2}\right)\right\}$.

Example 3 continued. For this example $\mathcal{E}=$ $\left\{(0,0),\left(k_{3} / k_{2}, k_{1} / k_{2}\right)\right\}$.

Example 4 continued. For this example $\mathcal{E}=$ $\left\{\left(x_{1}, x_{2}, x_{3}, x_{4}\right) \in \overline{\mathcal{R}}_{+}^{4}: x_{2}=0\right.$ and $\left.x_{1} x_{3}=0\right\}$. For the reduced system $(4.6),(4.7)$, if $x_{2}(0)+x_{3}(0)>0$ then $\mathcal{E}=\{(0,0)\}$, whereas if $x_{2}(0)+x_{3}(0)=0$ then $\mathcal{E}=\left\{\left(x_{1}, 0\right): x_{1} \geq 0\right\}$.

The following result given in [4] provides a sufficient condition for semistability.

Theorem 5.1. Assume that $\mathcal{U} \subseteq \mathcal{D}$ is closed and invariant with respect to $(5.1)$ and suppose that every trajectory with $x(0) \in \mathcal{U}$ of (5.1) is bounded. Furthermore, let $V: \mathcal{D} \rightarrow \mathcal{R}$ be a $\mathrm{C}^{1}$ function such that $V(x) \geq 0$ and $\dot{V}(x) \leq 0$ for all $x \in \mathcal{U}$. Finally, let $\mathcal{M}$ denote the largest invariant subset of $\{x \in \mathcal{U}: \dot{V}(x)=0\}$. If every element of $\mathcal{M}$ is a Lyapunov stable equilibrium with respect to $\mathcal{U}$, then every equilibrium $x_{\mathbf{e}} \in \mathcal{U}$ of (5.1) is semistable with respect to $\mathcal{U}$.

The following result uses the Lyapunov function $V(x)=\mu^{\mathrm{T}} x$ to analyze the stability of the zero solution of (2.3). Note that 0 is an equilibrium of (2.3) if and only if $A$ has no zero rows, that is, if and only if 0 is not a reactant of the reaction network (2.2).

Proposition 5.1. Assume 0 is an equilibrium of (2.3) and suppose there exists $\mu>>$ such that $B \mu \leq \leq A \mu$. 
Then 0 is Lyapunov stable with respect to $\overline{\mathcal{R}}_{+}^{s}$. If, in addition, $B \mu<<A \mu$, then 0 is globally asymptotically stable with respect to $\overline{\mathcal{R}}_{+}^{s}$.

Example 1 continued. Let $\mu=\left[1 / k_{1} 1 / k_{2}\right]^{\mathrm{T}}$ so that $(A-B) \mu=0$. It thus follows from Proposition 5.1 that 0 is Lyapunov stable. Since the kinetic equation is linear it follows that $M$ is both Lyapunov stable and semistable.

Example 2 continued. First note that because of the structure of the set of equilibria, none of the equilibria are asymptotically stable. Next we consider an equilibrium $x_{e}$ of the form $(0, \epsilon)$, where $\epsilon>0$. By linearizing the system about this equilibrium, it can be seen that this equilibrium is not Lyapunov stable. Hence it remains to determine the stability of an equilibrium of the form $\left(\delta, k_{2} \delta / k_{1}\right)$, where $\delta \geq 0$. To do this, let $\mathcal{U}$ be the closed set $\mathcal{U} \triangleq\left\{\left(x_{1}, x_{2}\right) \in \overline{\mathcal{R}}_{+}^{2}: x_{2}-a x_{1} \leq 0\right\}$, where $a>k_{2} / k_{1}$. Note that $\mathcal{U}$ is invariant since $d / d t\left(x_{2}-a x_{1}\right)$ is nonpositive on the set $\left\{\left(x_{1}, x_{2}\right): x_{2}=a x_{1}\right\}$. Note that all of the equilibria contained in $\mathcal{U}$ are of the form $\left(\delta, k_{2} \delta / k_{1}\right)$. Next, define the Lyapunov candidate $V: \mathcal{U} \rightarrow \mathcal{R}$ by

$$
V(x)=\frac{1}{2}\left(x_{1}-\delta+x_{2}-k_{2} \delta / k_{1}\right)^{2}+\frac{1}{2}\left(k_{1} x_{2}-k_{2} x_{1}\right)^{2} .
$$

Then, for all $\delta \geq 0$, we have $V\left(\delta, k_{2} \delta / k_{1}\right)=0$ and $V(x)>0$ for all $x \in \mathcal{U} \backslash\left\{\left(\delta, k_{2} \delta / k_{1}\right)\right\}$. Since $\dot{V}(x)=$ $-\left(k_{1}+k_{2}\right) x_{1}\left(k_{1} x_{2}-k_{2} x_{1}\right)^{2} \leq 0$ for all $x \in \mathcal{U}$ it follows that the equilibrium $\left(\delta, k_{2} \delta / k_{1}\right)$ is Lyapunov stable with respect to $\mathcal{U}$ for all $\delta \geq 0$. Finally, to show semistability, define $U(x)=x_{1}+x_{2}$, which satisfies $U(0)=0$, $U(x)>0, x \in \mathcal{U} \backslash\{0\}$, and $U(x)=0, x \in \mathcal{U}$. Hence every trajectory in $\mathcal{U}$ is bounded. Then $\dot{V}^{-1}(0)=f^{-1}(0)$, which shows that $\dot{V}^{-1}(0)$ is an invariant set. Thus the largest invariant set $\mathcal{M}$ contained in $\dot{V}^{-1}(0)$ is the set of equilibria $\left\{\left(\delta, k_{2} \delta / k_{1}\right): \delta \geq 0\right\}$ all of which are Lyapunov stable. Hence, by Theorem 5.1, the kinetic equation is semistable with respect to $\mathcal{U}$.

Example 3 continued. By linearizing the kinetic equation about the origin, it can be seen that the origin is not a Lyapunov stable equilibrium. To analyze the stability of the equilibrium $x_{\mathrm{e}}=\left(k_{3} / k_{2}, k_{1} / k_{2}\right)$, consider the function $U: \mathcal{R}_{+}^{2} \rightarrow \mathcal{R}$ defined by $U(x)=$ $k_{2}\left(x_{1}+x_{2}\right)-k_{3} \ln x_{1}-k_{1} \ln x_{2}$, which satisfies $\dot{U}(x)=0$ for all $x \in \mathcal{R}_{+}^{2}$. It can be seen from the form of the gradient and the Hessian of $U$ that $x=x_{\mathrm{e}}$ is an isolated local minimizer of $U$. Since $x_{\mathrm{e}}$ is the only stationary point in $\mathcal{R}_{+}^{2}$ it follows that $x=x_{\mathrm{e}}$ is also the global minimizer of $U$. Hence $V(x)=U(x)-U\left(x_{e}\right)$ satisfies $V\left(x_{\mathrm{e}}\right)=0$ and $V(x)>0$ for all $x \in \mathcal{R}_{+}^{2} \backslash\left\{x_{\mathrm{e}}\right\}$. Hence the equilibrium $x_{\mathrm{e}}=\left(k_{3} / k_{2}, k_{1} / k_{2}\right)$ is Lyapunov stable with respect to $\mathcal{R}_{+}^{2}$. Since the solutions consist of closed orbits, this equilibrium is not semistable.

Example 4 continued. For this example let $\mu=$ $\left[\begin{array}{llll}1 & 2 & 1 & 1\end{array}\right]^{\mathrm{T}}>>0$ so that $(A-B) \mu=0$. It thus follows from Proposition 5.1 that 0 is Lyapunov stable with respect to $\overline{\mathcal{R}}_{+}^{4}$. For the reduced kinetic equations (4.6), (4.7), assuming that $x_{2}(0)+x_{3}(0)>0$ it follows that $x_{1}=x_{2}=0$ is the only equilibrium. Now consider the radially unbounded Lyapunov function $V\left(x_{1}, x_{2}\right)=$ $\frac{1}{2} k_{3} x_{2}^{2}+\frac{1}{2} k_{1} \gamma_{1}\left(x_{1}+x_{2}\right)^{2}$. Since $\dot{V} \leq 0$ for all $x_{1}, x_{2} \geq 0$, global asymptotic stability follows from the invariant set theorem.

\section{The Zero Deficiency Theorem}

In this section we analyze the stability of positive equilibria of the kinetic equation (2.3) using the zero deficiency theorem [3]. A complex is either a reactant or a product. Let $m \geq 1$ denote the number of distinct complexes of the reaction network (including the reactant or product 0 if present), and denote the complexes by $c_{1}, \ldots, c_{m}$. We can identify each complex with a row of $A$ or $B$ so that $c_{i} \in \mathcal{R}^{1 \times s}$. Thus $m$ is the number of distinct rows of $\left[\begin{array}{l}A \\ B\end{array}\right]$.

Definition 6.1. Let $c_{i}$ and $c_{j}$ be complexes of the reaction network (2.2). Then $c_{i}$ and $c_{j}$ are directly linked if either $c_{i} \rightarrow c_{j}$ or $c_{j} \rightarrow c_{i}$. Furthermore, $c_{i}$ and $c_{j}$ are indirectly linked if there exist complexes $c_{i_{1}}, \ldots, c_{i_{p}}$ such that $c_{i}$ is directly linked to $c_{i_{1}}, c_{i_{1}}$ is directly linked to $c_{i_{2}}, \ldots, c_{i_{p}}$ is directly linked to $c_{j}$. Finally, $c_{i}$ and $c_{j}$ are linked if either $c_{i}=c_{j}, c_{i}$ and $c_{j}$ are directly linked, or $c_{i}$ and $c_{j}$ are indirectly linked.

The statement that complexes $c_{i}$ and $c_{j}$ are linked is an equivalence relation on the set of complexes. This relation induces a partitioning of the set of complexes into disjoint linkage classes. Let $\ell$ denote the number of linkage classes and denote these linkage classes by $\mathcal{C}_{1}, \ldots, \mathcal{C}_{\ell}$. Since the reactant and product in each reaction belong to the same linkage class, it follows that $\ell \leq r$.

As noted in Section 4, the rank of the reaction network $q=\operatorname{rank}(B-A)$ satisfies $q \leq \min \{r, s\}$. The following result provides a bound for $q$ that is sometimes better. Some additional notation will be needed. For $i=1, \ldots, \ell$, let $m_{i}$ denote the number of complexes in $\mathcal{C}_{i}$ so that $\sum_{i=1}^{\ell} m_{i}=m$. Furthermore, for convenience we order the complexes $c_{1}, \ldots, c_{m}$ so that $\mathcal{C}_{1}=$ $\left\{c_{1}, \ldots, c_{m_{1}}\right\}, \mathcal{C}_{2}=\left\{c_{m_{1}+1}, \ldots, c_{m_{2}}\right\}$, etc. Next, we reorder the reactions so that the first $r_{1}$ rows of $\left[\begin{array}{ll}A & B\end{array}\right]$ 
include the complexes in $\mathcal{C}_{1}$, rows $r_{1}+1, \ldots, r_{1}+r_{2}$ of $\left[\begin{array}{ll}A & B\end{array}\right]$ include the complexes in $\mathcal{C}_{2}$, etc. For $i=1, \ldots, \ell$, we define the rank $q_{i}$ of the linkage class $\mathcal{C}_{i}$ to be the number of linearly independent rows in the submatrix of $B-A$ comprised of the rows of $\left[\begin{array}{ll}A & B\end{array}\right]$ corresponding to the complexes in $\mathcal{C}_{i}$. Note that $q \leq \sum_{i=1}^{\ell} q_{i}$. Finally, for $i=1, \ldots, \ell$, it can be seen that $m_{i} \leq r_{i}+1$, and thus $m \leq r+\ell$.

Lemma 6.1. Let $i \in\{1, \ldots, \ell\}$. Then $q_{i} \leq m_{i}-1$. Furthermore, $q_{i}=m_{i}-1$ if and only if the complexes in $\mathcal{C}_{i}$ are the vertices of an $\left(m_{i}-1\right)$-dimensional simplex in $\widetilde{\mathcal{R}}_{+}$.

If $q_{i}=m_{i}-1$ then the linkage class $\mathcal{C}_{i}$ has full rank. We recall that an affine set is the translate of a subspace. Furthermore, the affine hull of a set $\mathcal{S}$ is the smallest affine set that contains $\mathcal{S}$. It can be seen that $\mathcal{C}_{i}$ has full rank if and only if the subspace parallel to the affine hull of $\mathcal{C}_{i}$ has dimension $m_{i}-1$

Proposition 6.1. $q \leq m-\ell$.

Definition 6.2. The deficiency $\delta$ of the reaction network $(2.2)$ is

$$
\delta \triangleq m-\ell-q
$$

It thus follows from Proposition 6.1 that the deficiency of a reaction network is a nonnegative integer. If the deficiency of a reaction network is zero, then the reaction network has zero deficiency.

Example 1 continued. For this reaction network, $m=2, \ell=1, q=1$, and thus $\delta=0$.

Example 2 continued. For this reaction network, $m=2, \ell=1, q=1$, and thus $\delta=0$.

Example 3 continued. For this reaction network, $m=6, \ell=3, q=2$, and thus $\delta=1$.

Example 4 continued. For this reaction network, $m=3, \ell=1, q=2$, and thus $\delta=0$.

Now define the matrix $C \in \mathcal{R}^{m \times s}$ whose rows are $c_{1}, \ldots, c_{m}$. Furthermore, let $\hat{A}, \hat{B} \in \mathcal{R}^{r \times m}$ be the matrices whose rows are unit coordinate vectors in $\mathcal{R}^{m}$ and which satisfy

$$
A=\hat{A} C, \quad B=\hat{B} C
$$

Theorem 6.1. Assume that the reaction network (2.3) has zero deficiency. Then every positive equilibrium of (2.3) is Lyapunov stable for all rate constants $k_{1}, \ldots, k_{r}$.
Definition 6.3. Let $c_{i}$ and $c_{j}$ be complexes. Then there exists a direct path from $c_{i}$ to $c_{j}$ if $c_{i} \rightarrow c_{j}$. Furthermore, there exists an indirect path from $c_{i}$ to $c_{j}$ if there exist complexes $c_{i_{1}}, \ldots, c_{i_{p}}$ such that $c_{i} \rightarrow c_{i_{1}}$, $c_{i_{1}} \rightarrow c_{i_{2}}, \ldots, c_{i_{p-1}} \rightarrow c_{i_{p}}$. Finally, there exists a path from $c_{i}$ to $c_{j}$ if there exists either a direct path or an indirect path from $c_{i}$ to $c_{j}$. The reaction network (2.2) is weakly reversible if, for all pairs of complexes $c_{i}, c_{j}$, the existence of a path from $c_{i}$ to $c_{j}$ implies the existence of a path from $c_{j}$ to $c_{i}$.

Proposition 6.2. Assume that the reaction network (2.3) has zero deficiency. Then (2.3) has at least one positive equilibrium if and only if it is weakly reversible.

Proposition 6.3. Assume that the reaction network (2.3) has zero deficiency and is weakly reversible. Then every positive stoichiometric compatibility class contains exactly one equilibrium. Furthermore,

$$
\mathcal{E}_{+}=\left\{x \in \mathcal{R}_{+}^{s}: \log x-\log x_{\mathrm{e}} \in \mathcal{S}^{\perp}\right\}
$$

Theorem 6.2. Assume that the reaction network (2.3) has zero deficiency and has at least one positive equilibrium. Then every positive equilibrium of (2.3) is semistable.

\section{References}

[1] D. S. Bernstein and D. C. Hyland, "Compartmental Modeling and Second-Moment Analysis of State Space Systems," SIAM J. Matrix Anal. Appl., Vol. 14, pp. 880-901, 1993.

[2] P. Erdi and J. Toth, Mathematical Models of Chemical Reactions: Theory and Applications of Deterministic and Stochastic Models, Princeton University Press, 1988.

[3] M. Feinberg, "The Existence and Uniqueness of Steady States for a Class of Chemical Reaction Networks," Arch. Rational Mech. Anal., Vol. 132, pp. 311370, 1995.

[4] S. P. Bhat and D. S. Bernstein, "Lyapunov Analysis of Semistability," Amer. Contr. Conf., pp. 16081612, San Diego, CA, June 1999.

[5] D. S. Bernstein and S. P. Bhat, "Lyapunov Stability, Semistability, and Asymptotic Stability of Matrix Second-Order Systems," ASME Trans. J. Vibr. Acoustics, Vol. 117, pp. 145-153, 1995. 